\title{
Intradural Extramedullary Metastasis of Small Cell Lung Cancer: A Case Report
}

\author{
Chang-Hyun Lee, Ki-Jeong Kim, Seung-Jae Hyun, Tae-Ahn Jahng, Hyun-Jib Kim \\ Department of Neurosurgery, Spine Center, Seoul National University Bundang Hospital, \\ Seoul National University College of Medicine, Seongnam, Korea
}

A 60-year-old man presented with para-anesthesia and a tingling sensation in the saddle area. Intradural extramedullary (IDEM) tumors in conus medullaris were observed by radiologic studies. The patient underwent laminoplastic laminotomy at the T12-L2 level and subtotal removal of the tumor because of the ill-defined margin. Postoperatively, hypesthesia was improved up to $50 \%$. The IDEM tumor revealed a metastatic cancer originated from small cell lung cancer (SCLC) by histologic examination, and additional studies showed multiple metastases including adrenal gland, brain, and bone. Clinicians need to consider IDEM metastasis when SCLC patients were complained of neurologic symptoms mimic paraneoplastic syndrome. The pathophysioloigy of IDEM metastasis may be not only tertiary drop metastasis, but also faster mechanisms such as direct invasion.

Key Words: Small cell lung cancer $\cdot$ Intradural extramedullary $\cdot$ Spinal cord neoplasm $\cdot$ Metastasis $\cdot$ Diagnosis

\section{INTRODUCTION}

Metastatic spine tumors are classified according to their anatomical distribution. Upwards of $95 \%$ of spinal metastases occurred in extradural space, whereas intradural extramedullary (IDEM) and intramedullary metastases are very rare, ${ }^{3,10)}$. Perrin and colleagues reported that only $5 \%$ of 200 consecutive cases of spinal metastasis are IDEM metastases ${ }^{7}$. Common origin of spinal IDEM metastases are originated from neurogenic tumor such as melanoma, lymphoma, and medulloblastoma ${ }^{1)}$. In analyzing IDEM metastases of non-neurogenic origin, the most frequent histological subtype was lung adenocarcinoma.

Small Cell Lung Cancer (SCLC) presently accounts for approximately $13 \%$ of all newly diagnosed lung cancer cases ${ }^{2}$. SCLC is a common tumor of intramedullary metastasis from lung cancer and often progresses to leptomeningeal carcinomatosis. However, IDEM metastasis from SCLC is extremely rare ${ }^{9,11)}$. The only

- Received: April 13, 2012 - Revised: September 4, 2012

- Accepted: September 26, 2012

Corresponding Author: Ki-Jeong Kim, MD, PhD

Department of Neurosurgery, Spine Center, Seoul National University

Bundang Hospital, Seoul National University College of Medicine, 300

Gumi-Dong, Bundang-Gu, Seongnam 463-707, Korea

Tel: +82-31-787-7166, Fax: +82-31-787-4059

Email: kijeong@snu.ac.kr

"The institutional review board of Seoul National University Bundang Hospital approved the study (B-1 103/123-902). one case of IDEM metastasis originated from SCLC was reported $^{5)}$. Intradural metastasis of systemic cancer take considerable time to metastasis because it is known to occur as tertiary drop metastases as the secondary lesions from brain metasta$\mathrm{ses}^{8)}$. The previously reported case of IDEM metastasis from SCLC also took 10 months ${ }^{5}$. We report a synchronously presented IDEM metastasis case originated from SCLC and investigate unusual pathogenesis which may be direct invasion.

\section{CASE REPORT}

A 60-year-old male presented with back pain, para-anesthesia, and a tingling sensation in the saddle area over the previous 6 months. His symptoms had gradually progressed. Neurological examination at admission demonstrated anesthesia below the T12 dermatome, and decreased rectal tone. The patient complained of chronic cough for 4 months and had a medical history of asthma diagnosed 2 months ago. He has been prescribed a bronchodilator. A preoperative chest roentgenogram did not show any mass-like lesion. Lumbar magnetic resonance (MR) images revealed an irregular marginated $1.4 \times 1.3 \times 1.7$ $\mathrm{cm}$ IDEM tumor at the conus medullaris (Fig. 1). The tumor was homogenously well-enhanced and multiple small-sized nodular enhancing lesions lay along the thecal sac from T12 to S1.

Subtotal removal of the tumor via laminoplastic laminotomy 
of T12, L1, and L2 was performed. The tumor showed a hypervascular, purplish, and friable mass (Fig. 2). It was not encapsulated and encased the conus and rootlets. There was no gliotic plane between rootlets and tumor, which was difficult to dissect it. Multiple small masses also adhered to many rootlets that could not be completely removed. Postoperatively, hypesthesia was improved up to $50 \%$. Histologic exam revealed that marked increased cellularity and moderate nuclear pleomorphism. Tumor cells are small round, oval and spindleshaped cells with scanty cytoplasm (Fig. 3). Immunohistochemical staining showed positive result for cytokeratin, vimentin, and synaptophysin. The sample was negative for thyroid transcription factor-1 and inhibin (Fig. 4). Hence, histological examination showed features of metastatic SCLC. Further studies including whole body positron emission tomography (PET)
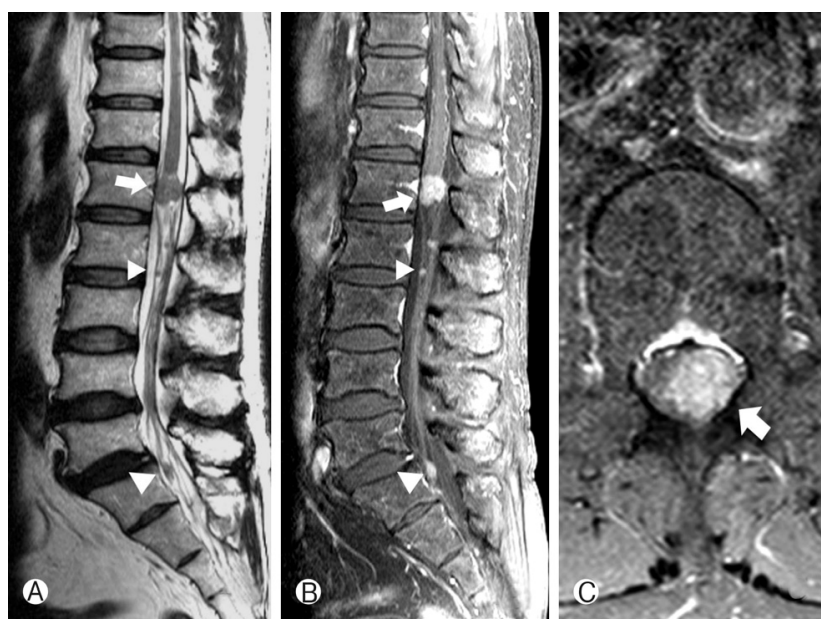

Fig. 1. Preoperative T2-weighed MR image showed iso-signal inten sity mass in L1,L2, and S1 level (A). Gadolinium enhanced T1weighed MR image showed well enhanced IDEM tumor (arrow) in L1 level. Multiple small tumors (arrow head) lay along the thecal sac (B). Spinal cord was deviated to the right by tumor. The margin of tumor was irregular and indistinguishable from thecal sac (C).

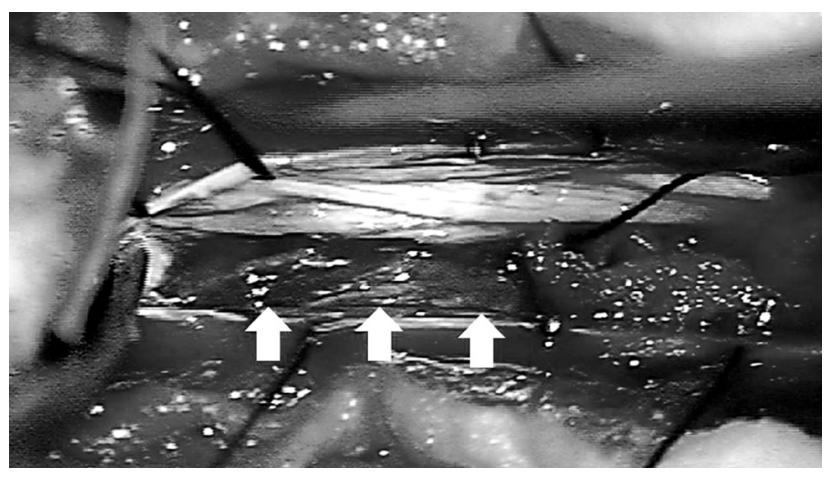

Fig. 2. Intraoperative photograph indicating the tumor, the intradural dark brown-colored mass (arrow), which adhered tightly to the rootlet. The tumor had a jelly like consistency and also hypervascularity. revealed multiple metastases (lung, adrenal gland, brain, and lymph node). SCLC located just behind the aorta and compressed right main bronchus (Fig. 5), which is misdiagnosed of asthma based on a false negative result by chest roentgenogram. The patient was treated with adjuvant chemoradiotherapy and maintained good condition till 9 months after surgery.

\section{DISCUSSION}

In the literature, five routes for metastatic intradural spinal tumor from the outside the central nervous system have been hypothesized: (1) the rich venous plexus; (2) perineural lymphatics $^{7}$; (3) seeding from involved osseous structures to the cerebrospinal fluid (CSF) through the dura; (4) spreading via

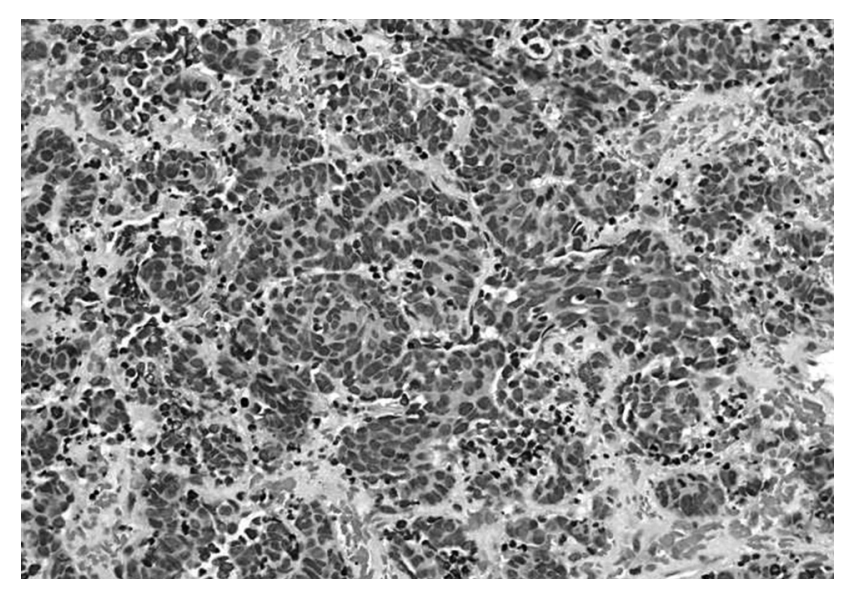

Fig. 3. A microphotograph indicates marked increased cellularity and moderate nuclear pleomorphism (Hematoxylin and eosin stain, $\times 400)$.

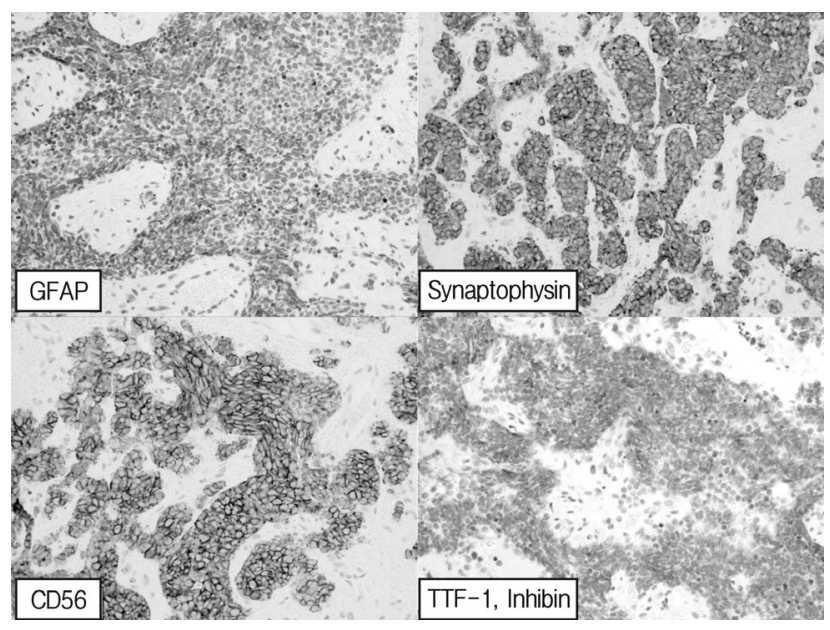

Fig. 4. Positive immunohistochemical staining for cytokeratin, vimentin, and synaptophysin. The sample was negative for thyroid transcription factor-1 (TTF-1) and inhibin. 
subarachnoid space; and (5) hematogenous spreading via the arterial system ${ }^{4}$. IDEM metastases are commonly thought to originate from CSF seeding. First, tumor cells are transferred to the brain (secondary drop metastasis), and then they enter the CSF and are transported throughout the nervous system by CSF flow. This spreading causes either multifocal or diffuse infiltration of the leptomeninges and is known as leptomeningeal carcinomatosis. Finally, metastatic tumors arise in IDEM lesions (tertiary drop metastasis). The mechanism via brain may take considerable time to detect IDEM.

In the literature review, the development of IDEM or intramedullary metastasis was divided into earlier and later cases. The delayed metastasis may be related with tertiary drop metastasis. However, early metastasis could not be explained by tertiary drop hypothesis. The mechanism of early intradural metastasis faster than tertiary drop metastasis may be present. Okamoto and colleagues studied a total of 1,215 cases of primary lung cancer at autopsy ${ }^{6}$. Ten cases of the patients revealed intramedullary involvement from SCLC. Comparing the interval between the diagnosis of SCLC and intramedullary metastasis in documented cases, the patients were divided two groups by metastasis interval. It takes mean 3.6 months of early detected five patients. The other patients were detected at 20.1 months.

In our case, the patient showed osteoblastic lesion of T11, which is just behind of lung cancer (Fig. 5). Both vertebral body of T11 and lamina also showed slightly increased uptake in PET scan. T11 bone metastasis lay on the direct connection between primary lesion and IDEM metastasis. Hence, one of possible pathogenesis is a direct tumor invasion. However, spinal metastasis seldom invades both intradural and extradural space. Further evaluation is needed about them. SCLC usually showed leptomeningeal seeding and intramedullary tumor in spinal lesion. Lin and colleagues reported the first IDEM metastasis of SCLC that caused cauda equina syndrome $e^{5}$. Their case was already diagnosed with SCLC one year ago and IDEM metastasis was developed as treatment failure. The patient in this paper complained of neurologic deficits as the initial symptoms, and SCLC was diagnosed by PET and chest CT after metastasis surgery. Despite prior 2 months of pulmonary symptom, IDEM metastasis of the patient was not accord with traditional mechanism as called tertiary drop metastasis.

SCLC is well known for a variety of paraneoplastic syndromes, which have vague and multi-systemic symptoms and can cause sensory changes or motor weakness. Therefore, patients with spinal metastases could be confused with only having paraneoplastic syndromes. Neurologic symptoms of the patient were also unusual. Neurologic deficits rapidly progressed within 6 months and became very severe. In the reported case review, some patients went to the hospital several weeks

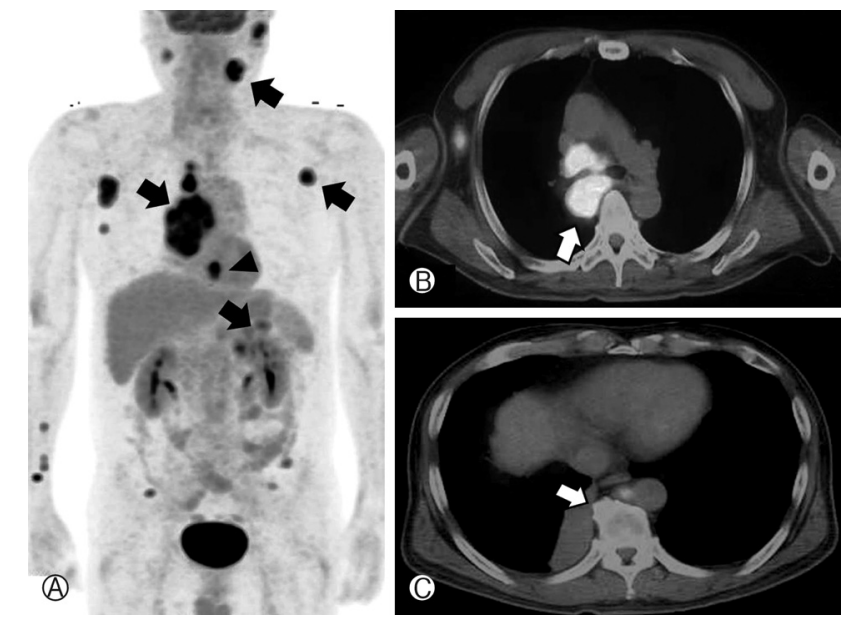

Fig. 5. Positron emission tomography revealed high 18-fluoro-2-deoxyglucose uptake masses indicating multiple metastatic lesions (arrow). Central-type lung cancer and axillary lymph node, parotid gland and adrenal gland metastases were diagnosed (A). Chest-enhanced computed tomography showing the right main bronchus that was compressed by the tumor (arrow) (B). Gadolinium-enhanced brain MR imaging. Note the homogenous multiple scattered enhancing lesions (arrow heads) (C).

or months after the onset of neurologic symptoms. What is worse, a few patients were treated with pain killers without the clinicians considering the possibility of metastasis ${ }^{5 \text {. }}$. Clinicians need to concern neurologic signs in aspect of metastasis.

\section{CONCLUSION}

This is the rare case report of IDEM metastasis in SCLC. IDEM metastasis may be not always followed the rule as known as tertiary drop metastasis. Hence clinicians need to consider IDEM metastasis when SCLC patients were complained of neurologic deficits mimic paraneoplastic syndrome.

\section{REFERENCES}

1. Chow TS, McCutcheon IE: The surgical treatment of metastatic spinal tumors within the intradural extramedullary compartment. J Neurosurg 85:225-230, 1996

2. Govindan R, Page N, Morgensztern D, Read W, Tierney R, Vlahiotis A et al: Changing epidemiology of small-cell lung cancer in the United States over the last 30 years: analysis of the surveillance, epidemiologic, and end results database. J Clin Oncol 24:4539-4544, 2006

3. Jacobs WB, Perrin RG: Evaluation and treatment of spinal metastases: an overview. Neurosurg Focus 11:e10, 2006

4. Kubota M, Saeki N, Yamaura A, Iuchi T, Ohga M, Osato K: A rare case of metastatic renal cell carcinoma resembling a nerve sheath tumor of the cauda equina. J Clin Neurosci 11:530-532, 2004 
5. Lin CL, Chang JL, Lo HC, Wu KA: Extramedullary-intradural spinal metastasis of small cell lung cancer causing cauda equina syndrome. Am J Med Sci 339:192-194, 2010

6. Okamoto H, Shinkai T, Matsuno Y, Saijo N: Intradural parenchymal involvement in the spinal subarachnoid space associated with primary lung cancer. Cancer 72:2583-2588, 1993

7. Perrin RG, Livingston KE, Aarabi B: Intradural extramedullary spinal metastasis. A report of 10 cases. J Neurosurg 56:835-837, 1982

8. Schick U, Marquardt G, Lorenz R: Intradural and extradural spinal metastases. Neurosurg Rev 24:1-5, 2001

9. Schiff D, O'Neill BP: Intramedullary spinal cord metastases: clinical features and treatment outcome. Neurology 47:906-912, 1996

10. Shin DA, Park SW, Kim KN, Yoon DH: Spinal leptomeningeal metastasis from recurrent cerebral glioblastoma. Korean J Spine 1:146-149, 2004

11. Vaporciyan AA, Kies MS, Stevens CW, Komaki R, Roth JA: Cancer of the lung, ed 5. Atlanta: BC Decker, pp1394-1406, 2005 